\title{
Control sample for galaxy pairs: Simulations and Observations
}

\author{
Josefa Perez ${ }^{1,2,3}$ and Patricia Tissera ${ }^{1,2}$ \\ ${ }^{1}$ Instituto de Astronomía y Física del Espacio,Conicet-UBA, CC67, Suc.28, \\ Buenos Aires, Argentina. email: jperez fcaglp.unlp.edu.ar \\ ${ }^{2}$ Facultad de Ciencias Astronomía y Geofísica, Universidad Nacional de La Plata, Argentina. \\ ${ }^{3}$ Consejo Nacional de Investigaciones Científicas y Técnicas, CONICET, Argentina.
}

\begin{abstract}
Several attempts have been made in order to isolate the effect of galaxy interactions by comparing galaxy in pairs with isolated galaxies. However, different authors have proposed different ways to build these control samples (CS). By using mock galaxy catalagues built up from the Millennium Simulation, we show that the set of constrains used to define a CS might introduce biases which could affect the interpretation of results. In this analysis, we make use of the fact that the physics of interactions is not included in the semi-analytic model, so that any difference between the mock control and pair samples can be attributed to selection biases. Thus, we suggest how to build an unique and unbiased CS in order to individualize the effect of interactions. Based on this theoretical findings and using the SDSS-DR4 data, we revise some previous observational results of galaxy in pairs in order to evaluate any possible disagreement. The comparison between simulations and observations suggests an overestimated effect of the DM halo bias in semianalytical models.
\end{abstract}

Keywords. galaxies: evolution, galaxies: interactions, surveys.

\section{Galaxy Pair and CS definitions}

From a mock galaxy catalague built up from the SAM of De Lucia and Blaizot (2007), and according to previous observational studies (Lambas et al. 2003), we build a Galaxy Pair (GP) Catalogue imposing thresholds in relative projected separations and radial velocities $\left(r_{p}<100 \mathrm{kpch}^{-1} ; \Delta V<350 \mathrm{kms}^{-1}\right)$. A basic CS is constructed by selecting galaxies without a near companion within these thresholds, and requiring that its members match one-to-one the redshift and r-band magnitude of the GP. As discussed in the next section, this CS definition is sequentially improved in order to remove the effects of a biased selection (also see Perez et al. 2009a).

\section{Results and Conclusions}

We analyse possible bias effects in the selection of a basic CS built for styding galaxy pairs. This study relies on the fact that the physics of interactions is not included in the SAM, so any difference in the porperties of the mock control and pair samples can be attributed to selection biases. We show that galaxy pairs tend to have slightly larger stellar masses and more concentrated morphologies when they are compared with isolated galaxies with the same redshift and r-band magnitude. Galaxy pairs also tend to inhabit more local dense regions and tend to be hosted by DM haloes of larger masses. These findings suggest that an unbiased CS should be constructed by requiring that its members match one-to-one the redshift, the stellar and halo masses, the morphology and the local projected density of each GP (Perez et al. 2009a). 
Analysis of the SDSS-DR4 data shows that contrary to simulations, the largest bias effect in the observational CS is introduced by the local density parameter, giving to the dark matter halo mass a less significant role in the CS definition. This, in fact, contributes to show an overestimated effect of the DM halo bias in the SAM, introduced by an exacerbated environmental treatment of satellite galaxies (Perez et al. 2009a).

Some previous observational results of galaxy pairs are revised in order to determine how the corrections in the CS definition could affect the interpretation of results (Alonso et al. 2006; Michel-Dansac et al. 2008; Ellison et al. 2008). We analyse the colour distribution and mass-metallicity relation of GP, using for comparison the unbiased CS theoretically suggested (Perez et al. 2009a). Our result shows that after removing the bias selection in the CS, previous conclutions about the effect of galaxy interaction on colours and metallicities are confirmed. However, a correct definition of the CS seems to be decisive when the SF-Environment relations are analysed. GP tend to have an excess of passive star forming systems inhabiting high density regions when are compared with galaxies in the basic CS. This excess is reduced if we consider the unbised CS, indicating an strong effect of biases (DM halo and local density environment) and a more relative role of interactions in driving this relation (Perez et al. 2009b).

\section{References}

Alonso, M. S., Lambas, D. G., Tissera, P., \& Coldwell, G. 2006, MNRAS 367, 1029

De Lucia, G. \& Blazoit, J. 2007, MNRAS 375, 2

Ellison, S. et al. 2008, AJ 135, 1877

Lambas, D. G., Tissera, P. B., Alonso, M. S., \& Coldwell, G. 2003, MNRAS 346, 1189

Michel-Dansac, L., Lambas, D. G., Alonso, M. S., \& Tissera, P. 2008, MNRAS 386, 82

Perez, J., Tissera, P., \& Blaizot, J. 2009, MNRAS 397, 748 (Paper a)

Perez, J., Tissera, P., Padilla, N., Alonso, S., \& Lambas, D. G. 2009, in press by MNRAS (Paper b, astroph/0904.2851) 